\title{
Parameter identification of nonlinear time-dependent rubber bushings models towards their integration in multibody simulations of a vehicle chassis
}

\author{
Guillaume Puel ${ }^{1}$, Béatrice Bourgeteau, Denis Aubry \\ Laboratoire MSSMat, Ecole Centrale Paris / CNRS UMR 8579, Grande Voie des \\ Vignes, 92290 Châtenay-Malabry, France
}

\begin{abstract}
Rubber bushings are extensively-used linking parts in a vehicle chassis that allow to filter noise and vibration. They influence much, however, the transient behaviour of the vehicle, such as its steering performance. Therefore building a multibody simulation with a relevant description of the rubber bushings is useful to describe the significant characteristics of the vehicle's steering behaviour. First, a nonlinear time-dependent model describing a rubber bushing's mechanical behaviour is presented. In order to be relevant, the parameters associated with this model are then identified from experimental tests using an adjoint state formulation of the identification problem. The identified values are eventually validated using additional experimental data.
\end{abstract}

Keywords: rubber bushings, nonlinear time-dependent model, parameter identification, adjoint state, multibody simulation

\section{Introduction}

Rubber bushings are mechanical parts that are extensively used to reduce

noise and vibration in a vehicle chassis. They have, however, a strong im-
pact on the whole vehicle chassis behaviour, such as its steering performance.

Email addresses: guillaume.puel@ecp.fr (Guillaume Puel), beatrice.bourgetau@sagem.com (Béatrice Bourgeteau), denis.aubry@ecp.fr (Denis Aubry)

${ }^{1}$ Corresponding author. 
Since it is not always possible to get experimental data directly on the whole chassis system, their impact has to be described using a multibody simulation. This would include a relevant model of the rubber bushings mounted in the chassis. This paper focuses on the identification procedure that allows to determine the parameters associated with such a model. This will be done by means of experimental data derived from specific measurements on bushings mounted in test rigs.

The following provides an outline of the paper. First, the rubber bushings' behaviour enlightening the different phenomena to take into account in the model is briefly presented. Then the characteristics of the chosen model are given; two points are underlined: how relevant this choice is to describe the observed phenomena, and how the results coming from the associated multibody simulations allow to describe some qualitative, but significant characteristics of the vehicle's steering behaviour. In order to derive more accurate, quantitative results from these simulations, the characterization of the actual rubber bushings' behaviour is needed. Consequently, a series of measurements on test rigs have been performed in conjunction with several suppliers of bushings. These tests are primarily used as an experimental reference in the identification procedure. This is the core of the study, which is described in detail: a misfit function expressing the discrepancy between the model's results and the experimental data is defined and then minimized by means of a gradient-based minimization algorithm using an adjoint state formulation. The identification results are eventually presented and discussed.

\section{Mechanical behaviour of the rubber bushings}

\subsection{Description of the mechanical part}

Rubber bushings are cylindrical parts that consist of a metallic inner core and a plastic or metallic outer frame linked one to the other by a rubber intermediate body (Figure 1). The inner core and the outer frames are connected to different parts of the chassis.

The geometry of the rubber intermediate body is not exactly axisymmetric: pits and ribs allow distinct behaviours for different load directions. Thus the bushing's tridimensional mechanical behaviour can be split into several simple uniaxial behaviours, corresponding to classical types of loads (axial, radial, torsional, ...). These different behaviours can be described in a simple way, for they can be directly related to the material behaviour of 


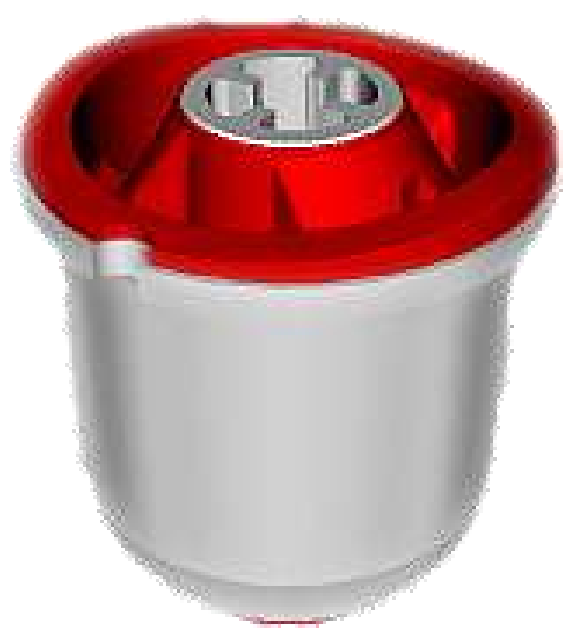

Figure 1: Typical rubber bushing

rubber. Thus in the following, the study of the mechanical characteristics of rubber will be studied rather than the influence of the geometry of the rubber intermediate body.

\subsection{Material mechanical behaviour of rubber}

Rubber usually exhibits three canonical components in its mechanical behaviour: an elastic component, which is totally reversible, and two dissipating (thus irreversible) components, which are hysteresis and viscoelasticity. Additional specific effects also have to be taken into account. All these behaviours have been listed in numerous references $[1,2,3]$, which tried to propose some associated simple models as the basis of more complex models.

The elastic component is characterized by a nonlinear stress versus strain curve, which can be obtained from cyclic measurements involving high-amplitude displacements at low speed: in this case, the irreversible effects can be neglected. If not, one way to derive the stress versus strain curve is to flatten the obtained cycle into an average curve assumed to represent the elastic component only. Furthermore, for rather small displacements, as it is generally the case for the bushings typically used, the stress versus strain relation can be considered as linear, or, at worst, as piecewise linear.

The hysteretic component is mainly due to the presence of carbon black as a reinforcing filler in rubber: inner friction-like phenomena due to these 
particles create a history effect, which depends on the loading speed. This component is of utter importance for the vehicle chassis behaviour, especially at small amplitudes where hysteresis is the most significant effect.

Carbon black particles also have an additional impact on the bushing's secant stiffness, called the Payne effect [4]. The secant stiffness is considered when plotting the force versus displacement curve as cyclic displacements are applied to the bushing, and is defined as the slope of the secant line joining the two extreme points of the obtained hysteresis cycle. For small displacement amplitudes, the Payne effect consists in a secant stiffness that decreases rapidly as the loading amplitude increases. This effect is significant enough to be taken into account in the bushings typically used: it can be seen in experimental measurements that the higher the proportion of carbon black fillers, the stronger this effect.

Whereas hysteresis is often a rate-independent phenomenon, viscoelasticity on the contrary depends on the loading speed, and can be related to the friction that takes place between the rubber macromolecules. The associated effects are particularly significant in relaxation, which occurs when a displacement characterised by a Heaviside step function is applied to the bushing: the force versus time curve depends on the amplitude of the displacement step function as well as its speed (for the application of the displacement is not truly discontinuous). Relaxation has to be taken into account because it denotes an evanescent memory effect, which is crucial in the vehicle's steering behaviour: two successive steering wheel rotations will not have the same effect depending if they are in the same direction or in opposite directions.

Another significant impact of the viscoelastic component can be observed in the frequency domain: there is a dynamic stiffening of the bushing as the loading frequency increases. The bushing's dynamic stiffness is derived from a first-harmonic analysis of the force obtained when a sinusoidal displacement is applied to the bushing: it is defined as the amplitude ratio of the force's (predominant) first harmonic to the displacement. This effect is opposed to the Payne effect: even if it is more limited, it should be taken into account in the bushing's model.

One last effect related to the mechanical behaviour of rubber should be evoked: the Mullins effect [5], which consists in a strong decrease in the bushing's secant stiffness occurring whenever the load increases beyond its prior all-time maximum value. After a few cycles at a given amplitude, it can then be assumed that the stiffness does not evolve any more, provided there is no applied load of higher amplitude. This damage-like effect, though 
significant, will not be taken into account here, for it is assumed that all the bushings which are used in the chassis are first exerted in a burn-in process: they are submitted before operational service to various loads along different directions, so that the Mullins effect is no longer significant once the bushings have been mounted on the chassis.

\section{Mechanical model for the rubber bushings}

Since the typical geometry of a rubber bushing is designed such that the mechanical behaviours along the different directions are decoupled, the geometry influence is not further studied, and a simple model can be proposed for each loading mode. Furthermore, the model should be chosen as simple as possible so that its implementation in a multibody simulation software could be easy and robust. Therefore rheological models are used that have to represent the different components described in Section 2.2. The degrees of freedom (DOFs) associated with these models correspond to the nodes linking the different rheological components. They are the solutions of the Ordinary Differential Equations (ODEs) associated with the chosen models.

\subsection{Standard Triboelastic Solid model (STS)}

The Standard Triboelastic Solid model (STS), initially proposed by Coveney [6] for seismic rubber bushings, is based on the linear superposition of a pure elastic behaviour (described by a spring element) and a hysteretic component (described by a solid friction element): the resulting combination, depicted in Figure 2, is the STS elementary cell, whose combination in series can represent a typical hysteretic cycle, for the different friction elements allow to reproduce the desired memory effect. The parameters associated with the STS elementary cell are the stiffness $K_{r}$ of the spring element and the threshold force $F_{f}$ of the friction element, which means that the friction element slides if and only if the norm of the load acting on it is higher than the specified threshold $F_{f}$.

Eventually, the proposed STS model, shown in Figure 3, is based on the serial combination of several identical STS elementary cells, along with two additional springs, $K_{0}$ and $K_{p a}$, standing for a global elastic stiffness and an additional stiffness for low-amplitude displacements respectively. The STS elementary cells are chosen identical for the sake of simplicity. Consequently, the proposed STS model is based on four distinct parameters, for the number 


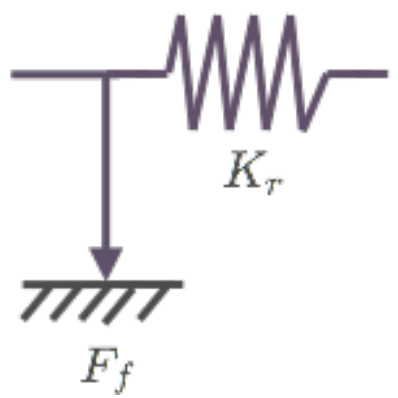

Figure 2: STS elementary cell

of STS elementary cells can generally be set once and for all. This number, indeed, allows a more or less fine discretization of the hysteresis cycle.

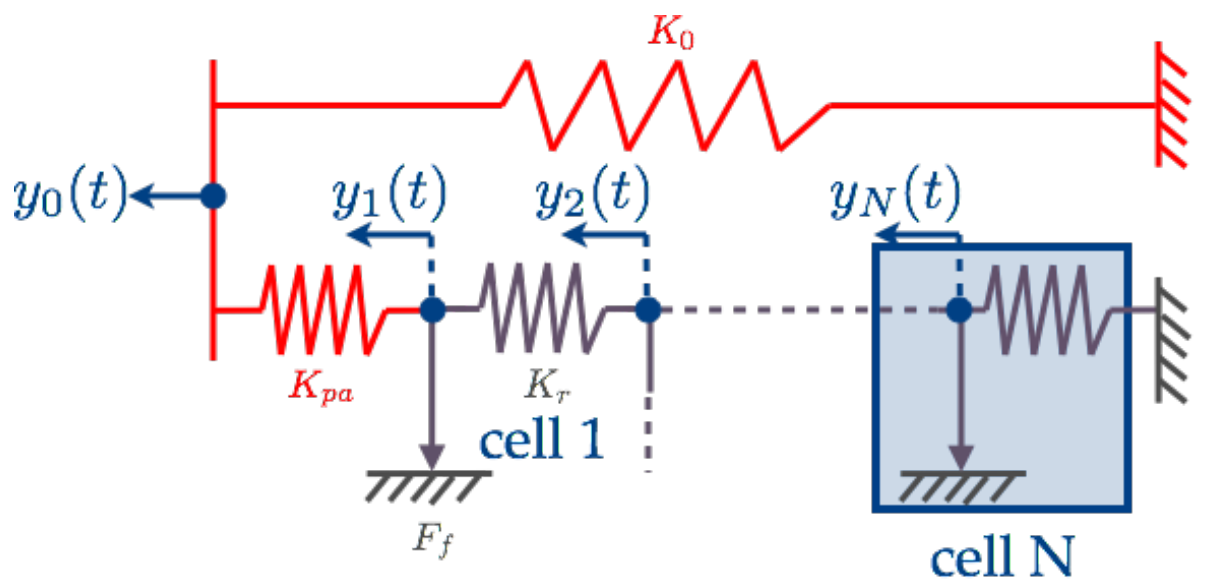

Figure 3: STS model with $N$ identical STS elementary cells

Although the STS model is able to represent the hysteretic component of the rubber bushing, it does not account for viscoelasticity. To take it into account, most current models add in parallel to the STS model elements with viscoelastic behaviour. For example, a Maxwell cell (i.e. viscous damping) is added in parallel in [7], but it generally seems difficult to suit the bushing's global dynamical response: [8] shows that using a viscous damping tends to entail an overestimation of the efforts in play. 


\subsection{Rate-dependent Triboelastic model (RT)}

Rather than add other components to the previous model, a better solution seems to replace the friction elements of the STS model by components exhibiting a viscoelastic behaviour: this is the Rate-dependent Triboelastic model (RT), proposed by Coveney as well [7]. The RT elementary cell comprises a linear spring element with stiffness $K_{r}$ and a nonlinear viscous dashpot put in parallel. The force versus velocity law of the dashpot is characterized by two parameters $\left(C_{0}, \alpha\right)$ such that:

$$
F_{k}(t)=C_{0} \operatorname{sign}\left(y_{k}^{\prime}(t)\right)\left|y_{k}^{\prime}(t)\right|^{\alpha}
$$

where $F_{k}(t)$ is the force applied on the dashpot and $y_{k}^{\prime}(t)$ is the velocity of the corresponding DOF. $\alpha$ is chosen such that $0<\alpha<1$ : depending on this value, the dashpot exhibits different behaviours. In particular, if $\alpha=0$, the dashpot is exactly equivalent to the solid friction element used in the STS model (when $C_{0}=F_{f}$ ).

Eventually, the proposed RT model is based on the serial combination of several identical RT elementary cells, along with two additional springs, $K_{0}$ and $K_{p a}$, standing for a global elastic stiffness and an additional elastic stiffness for low-amplitude displacements respectively. The RT elementary cells are chosen identical again for the sake of simplicity, resulting in the assembly depicted in Figure 4.

Since, once again, the number $N$ of RT elementary cells is set a priori (allowing a more or less fine discretization of the hysteretic cycle), the proposed RT model depends on $P=5$ distinct parameters: $\mathbf{p}=\left(\begin{array}{lll}K_{0} K_{p a} & K_{r} C_{0} \alpha\end{array}\right)^{\mathrm{T}}$. The forward problem thus consists in solving the following system of $N$ timedependent ODEs:

$$
\begin{gathered}
\left(K_{p a}+K_{r}\right) y_{1}(t)-K_{r} y_{2}(t)+C_{0} \operatorname{sign}\left(y_{1}^{\prime}(t)\right)\left|y_{1}^{\prime}(t)\right|^{\alpha}=K_{p a} y_{0}(t) \\
\vdots \\
-K_{r} y_{k-1}(t)+2 K_{r} y_{k}(t)-K_{r} y_{k+1}(t)+C_{0} \operatorname{sign}\left(y_{k}^{\prime}(t)\right)\left|y_{k}^{\prime}(t)\right|^{\alpha}=0 \\
\vdots \\
-K_{r} y_{N-1}(t)+2 K_{r} y_{N}(t)+C_{0} \operatorname{sign}\left(y_{N}^{\prime}(t)\right)\left|y_{N}^{\prime}(t)\right|^{\alpha}=0
\end{gathered}
$$

for $1<k<N$. The different DOFs $\left(y_{k}(t)\right)_{1 \leq k \leq N}$ are the components of the state vector $\mathbf{y}(t)$, whereas $y_{0}(t)$ is the prescribed displacement. It is also assumed that the initial conditions for each DOF are equal to zero. 


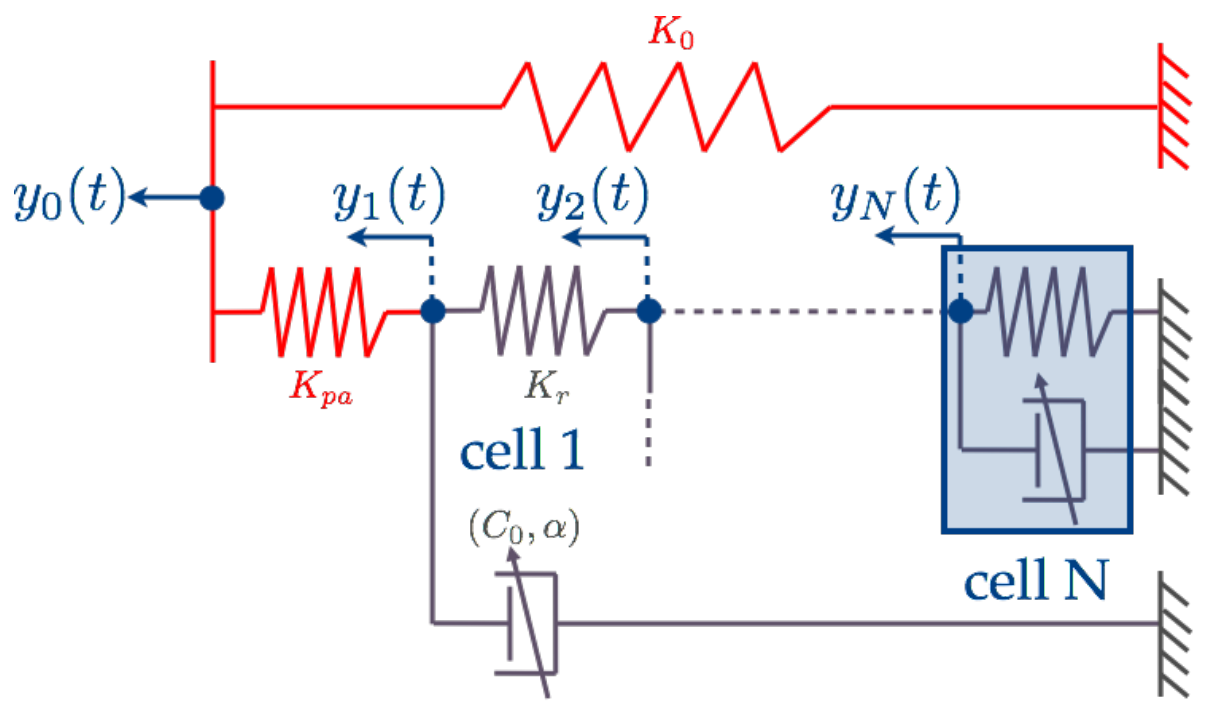

Figure 4: RT model with $N$ identical RT elementary cells

\subsection{Other models}

In addition to these two previous models, numerous models relying on alternative choices of rheological components have been proposed and extensively studied. For example, a fractional derivative model can be introduced in the viscoelastic part [9]. This kind of model, used in [10] along with a generalized friction force element, allows to accurately account for the evolutions of the dynamic stiffness of rubber isolators as the frequency and amplitude of the excitation, as well as the static precompression, evolve. This model, however, seems hardly suited to be implemented in a multibody simulation, because most of the associated algorithms used to solve the fractional derivative part in the time domain are rather sophisticated [11], or can be very time-consuming. Furthermore, the parameter identification seems far more awkward in the time domain than in the frequency domain.

Another widely-used model coming from [12] then [13] is the Bouc-Wen model consisting in a parametric hysteretic loop along with a spring and a linear viscous dashpot in parallel. The identification of the parameters associated with this model has been achieved in [14] in the frequency domain using a harmonic balance approximation. Used as is, however, the Bouc-Wen model exhibits some inconsistencies when compared with experimental data, and tends to overestimate the rate-independent hysteretic part relatively to 
the rate-dependent part. Several modifications have been introduced to improve the model, such as in [15] recently: the proposed model consists in two simple Bouc-Wen models put in parallel along with a spring and a linear viscous dashpot. Even if this modified Bouc-Wen model gives better predictions of the bushing's behaviour, it depends on 16 independent parameters. This makes the parameter identification rather difficult: in [15], a genetic algorithm is proposed, but the identified values of the different parameters strongly depend on the experimental data used in the identification process, because the inverse problem is significantly ill-posed due to the high number of parameters. The authors then propose to combine several experimental test results to improve the identification, but the process eventually seems to be delicate to apply.

\subsection{Simulations examples using the RT model}

Considering the conclusions of the previous section, the RT model seems to be the most relevant choice. First the number of RT cells has to be set so that the predictions are sufficiently refined to be similar to the curves obtained with the available measurements. Typically $N$ can be directly related to the discretization of hysteresis cycles as far as the simulation of quasistatic periodic displacements is concerned: Figure 5 shows the hysteresis cycles obtained for different values of $N$. The different parameters $\left\{K_{0}, K_{p a}, K_{r}, C_{0}, \alpha\right\}$ have been chosen such that the simulated cycles have similar features, i.e. the same values of the maximum load, the secant stiffness, and the tangents before and after cusps. For $N \geq 10$, the obtained curves have converged to a limit cycle whose smoothness seems satisfactory. Finally, $N=15$ cells are chosen to be able to account for applied displacements which would be larger than the ones proposed in the measurements, which could possibly happen in the vehicle chassis in operation.

In Figure 6, different simulations using the proposed RT model with $N=15$ elementary cells are depicted and show the model's ability to grasp most of the effects described in Section 2.2. On the left of Figure 6, the force-displacement curves, obtained when sinusoidal displacements are applied to the bushing, are plotted: they clearly show the bushing's stiffening as the loading frequency increases. More precisely, the secant stiffness, defined as the ratio between the maximum load and the maximum displacement, increases by $4 \%$ between $1 \mathrm{~Hz}$ and $10 \mathrm{~Hz}$, and by $4.5 \%$ between $10 \mathrm{~Hz}$ and $100 \mathrm{~Hz}$. On the right of Figure 6, one shows the force obtained when a displacement described by a Heaviside step function is applied to the bushing: 


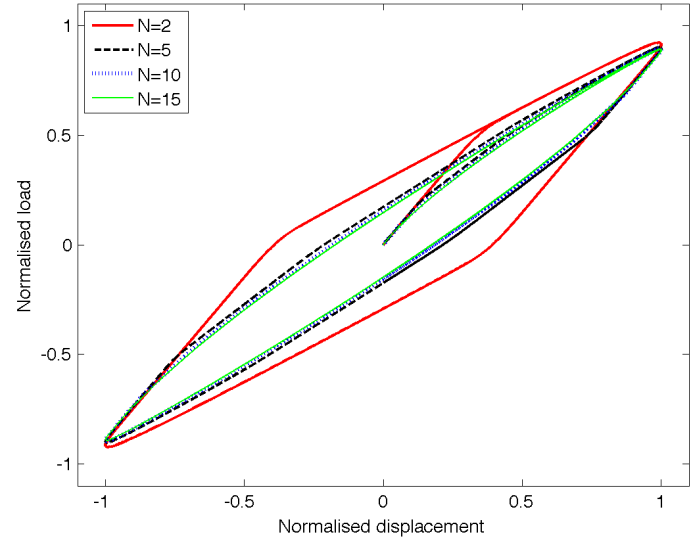

Figure 5: Numerical simulations obtained with the RT model for different numbers of cells

relaxation is clearly observed, until the force goes down to a given constant value, consistent with the bushing's static behaviour and the value of the applied steady-state displacement. In the same way, it is possible to verify that the proposed RT model is able to account for the Payne effect as well.
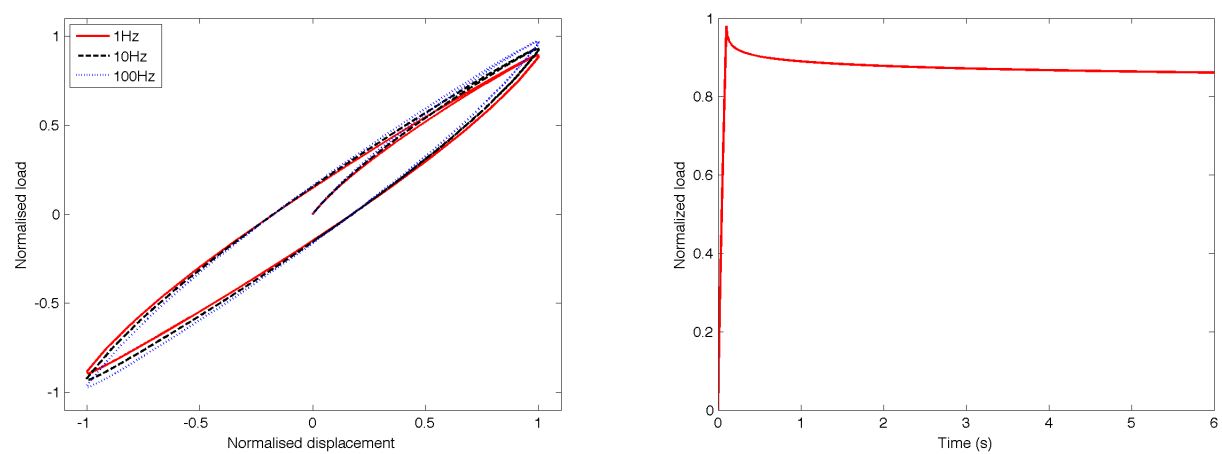

Figure 6: Numerical simulations obtained with the RT model for different prescribed displacements: sines of increasing frequencies (left) and Heaviside step function (right)

\subsection{Implementation in a multibody simulation code}

The RT model has then been implemented in a multibody simulation of a typical chassis using ADAMS software. More precisely, user functions 
programmed in Fortran allow to compute the load associated with the chosen model when a given displacement is applied to the rubber bushing.

Two different strategies are available. Using a GSESUB user function allows to solve the ODEs associated with the RT model along with the remaining equations associated with the whole multibody system. On the contrary, when a VARSUB user function is used, one has to implement directly in the subroutine the time integration algorithm. These two strategies have been tested on the very simple model of a beam linked to the ground by a rubber bushing whose mechanical behaviour is described by the RT model. They lead to similar results in terms of simulation results and accuracy. Nevertheless the VARSUB function seems to have more advantages than the GSESUB function: it is possible to implement any time integration scheme, independently from the one used for the remaining elements of the structure, and the computation time tends to be shorter. The final choice has then consisted of an explicit Euler scheme defined in the VARSUB function. The time step is chosen accordingly to the variable time step used by ADAMS, by dividing this latter by a given factor, which allows to guarantee the stability of the Euler time integration scheme.

Different simulations have been run on a complete multibody model of a typical small family car using RT models for the rubber bushings mounted in the front and rear axles of the vehicle. Only two specific results are shown here, and are associated with one specific rubber bushing, mounted in the rear axle, as depicted in Figure 7 . This rubber bushing, which links the rear crossbeam to the body, allows to filter vertical vibrations as well as to transmit longitudinal forces to the axle.

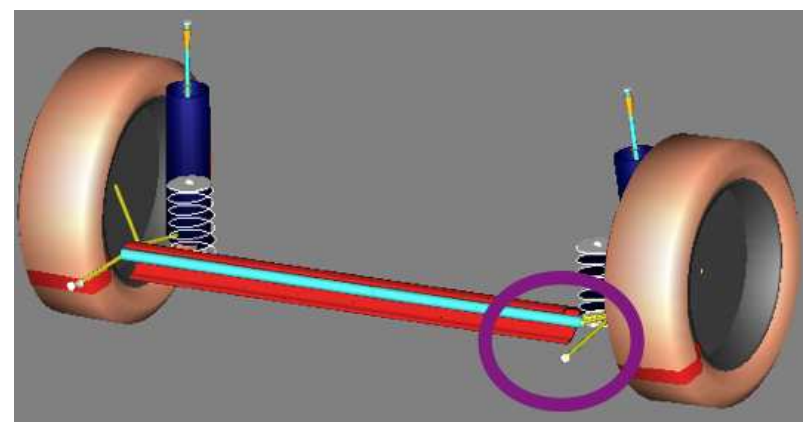

Figure 7: Location of one specific rubber bushing in the rear axle (partial view of the multibody model of a typical small family car) 
A first simulation focuses on how the studied bushing behaves when an angle characterised by a Heaviside step function is applied to the steering wheel of the vehicle (actually, the angular input is not truly discontinuous, but is characterised by a short time interval of constant angular velocity). Figure 8 shows the radial horizontal load in the bushing with respect to time in two different cases, depending on whether a RT model or a STS model is used to describe the rubber bushing's behaviour. The two curves are normalised by the respective maximum load values, which allows to easily compare the two models. This comparison stresses the lack of relaxation when using the STS model, whereas the RT model is able to account for this effect, as expected.

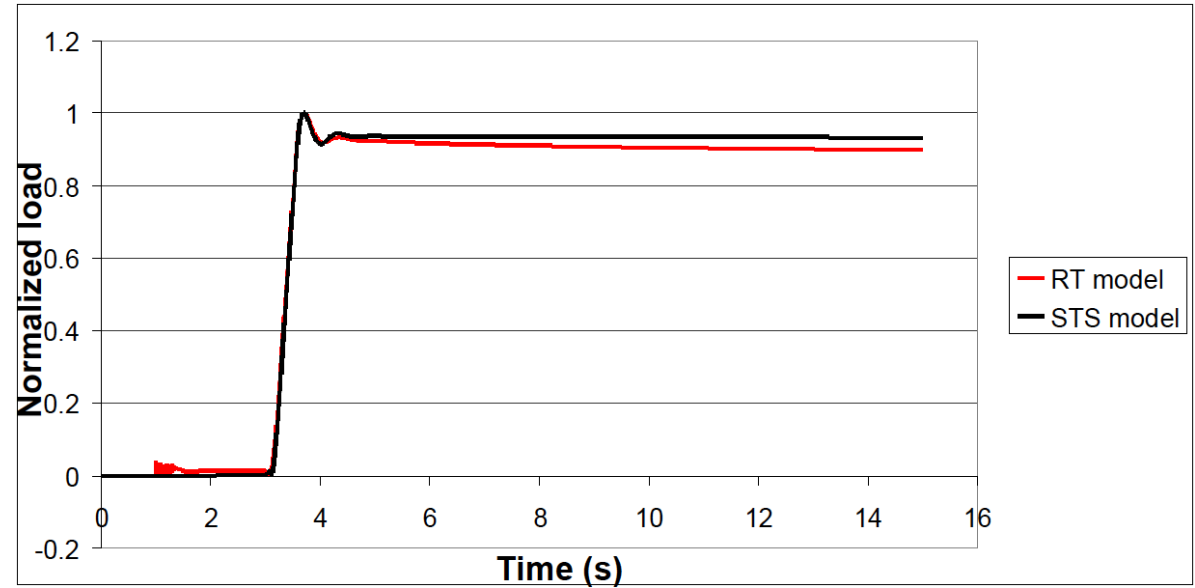

Figure 8: Normalised load in the bushing with respect to time (angular Heaviside step function on the steering wheel): RT model (red) and STS model (black)

A second simulation allows to represent well-known phenomena in the steering behaviour such as the evanescent memory effect. On the right of Figure 9 the (normalised) load acting on the studied bushing is shown, when two successive rotations (each described by a Heaviside step function) are applied to the vehicle's steering wheel. This kind of input is inspired from classical elk tests, consisting in avoiding an obstacle without braking and then coming back to the right lane. Depending on whether these two rotations are of the same direction or occur in opposite ways (as formally depicted on the left of Figure 9), the load acting on the bushing can be very different: the relaxation effect is stronger in the second case. This has an impact 
on the toe and camber angles of the wheel associated with the bushing of interest. Similar results can be obtained for other bushings, allowing to conclude on the impact on the vehicle's steering behaviour, which is a wellknown phenomenon described by test drivers.
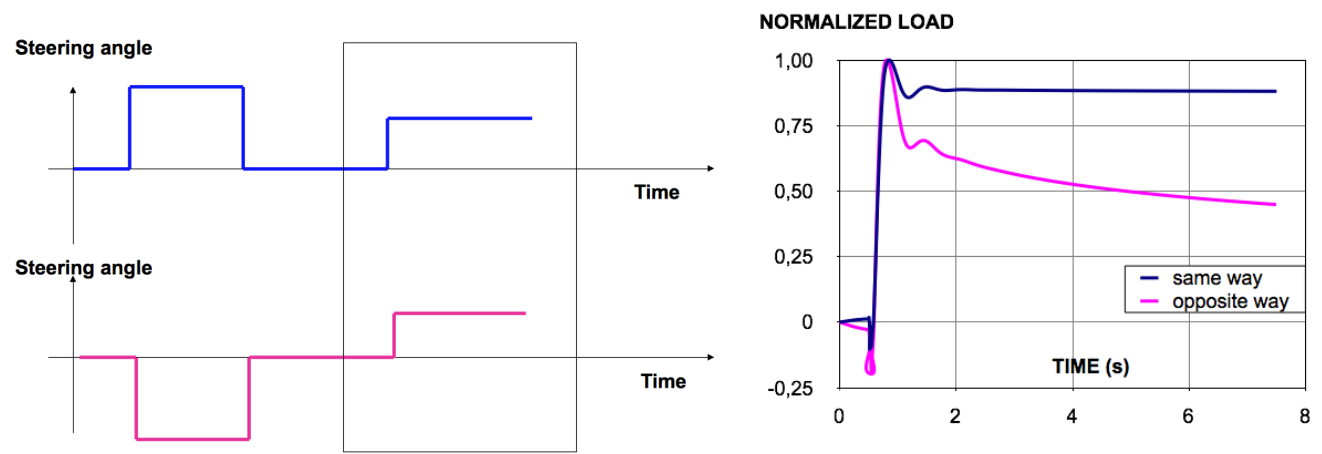

Figure 9: Normalised load during the second of two successive rotations of the vehicle's steering wheel: (blue) rotations of the same direction (pink) rotations of opposite directions (a positive rotation is equivalent to a left turn of the steering wheel)

The previous examples then demonstrate the relevance of the results predicted by the multibody simulation. However, without experimental data, which would be directly measured on the whole vehicle on the track, these predictions remain essentially qualitative. In order to turn these results into quantitative predictions, the next step consists in finding the correct values for the RT model's parameters. Since experimental information is available for each rubber bushing (coming from specific measurements in test rigs), the values of these parameters should be identified so that the response of a bushing, which is calculated using the RT model under a given loading mode, fits best the experimental measurements obtained with the same loading mode.

\section{Generic formulation of the identification problem}

For the sake of clarity, the identification problem as it is defined and solved is first presented in a generic way. The parameter identification of the RT model of rubber bushings is developed in the next section. 


\subsection{Generic formulation of the identification problem}

The forward problem (2) associated with the RT model can be described by the following implicit vector formulation of size $N$ :

$$
\begin{aligned}
& \mathcal{F}\left(\mathbf{y}(t), \mathbf{y}^{\prime}(t), \mathbf{p}, t\right)=\mathbf{0} \quad \forall t \in[0, T] \\
& \mathbf{y}(0)=\mathbf{0}
\end{aligned}
$$

where $\mathbf{p}$ of size $P$ and $\mathbf{y}$ of size $N$ are, just as before, the parameter vector and the state vector comprising all the DOFs of the forward model respectively.

For the identification problem, it can then be assumed that the experimental data will be compared with quantities derived from the different DOFs of the forward problem by means of a projection operator denoted $\mathbf{A}$, of size $n \times N$, where $n$ is the number of experimental measurements. Similarly, it is assumed that the experimental data can be written as if they were derived from an experimental state vector of size $N$ : this assumption has no other consequence than allowing a clearer presentation of the identification problem. Eventually, a so-called misfit function is defined, which expresses the discrepancy between the $n$ measurements $\mathbf{A y}_{\text {exp }}(t)$ and the $n$ associated quantities derived from the model $\mathbf{A y}(t ; \mathbf{p})$ :

$$
\mathcal{J}(\mathbf{p})=\frac{1}{2} \int_{0}^{T}\left|\mathbf{A}\left(\mathbf{y}(t ; \mathbf{p})-\mathbf{y}_{\exp }(t)\right)\right|^{2} \mathrm{~d} t+\frac{1}{2}\left|\mathbf{R}\left(\mathbf{p}-\mathbf{p}_{0}\right)\right|^{2}
$$

where $\mathbf{y}(t ; \mathbf{p})$ is the solution of the state equation (3) calculated with parameters $\mathbf{p}$. Since the forward problem is nonlinear, it seems relevant to use a time formulation of the misfit function, hence the integral over time.

The last term of the misfit function is a norm introduced to regularize the inverse problem, by expressing that the sought parameters $\mathbf{p}$ should be close to a vector $\mathbf{p}_{0}$ of typical values, with $\mathbf{R}$ a diagonal operator whose values are to be set. This so-called Tikhonov's regularization term aims at reducing the ill-posedness of the identification process by bounding the magnitude of the sought parameters.

\subsection{Minimization of the misfit function}

The identification problem consists in minimizing the misfit function (4) to find the parameters allowing the model to be as close to the experimental data as possible. This minimization is usually performed by means of gradient-based techniques, where the derivative of $\mathcal{J}(\mathbf{p})$ is analytically introduced using an adjoint state problem. This method was described in [16], 
which deals with the identification of elastoplastic constitutive laws, whose form is quite similar to the kind of law used in the RT model.

The solution $\mathbf{z}(t)$ of this adjoint state problem can be considered as a Lagrange multiplier introduced in the following Lagrangian function $\mathcal{L}(\mathbf{y}, \mathbf{p}, \mathbf{z})$ :

$$
\begin{aligned}
\mathcal{L}(\mathbf{y}, \mathbf{p}, \mathbf{z})= & \frac{1}{2} \int_{0}^{T}\left|\mathbf{A}\left(\mathbf{y}(t)-\mathbf{y}_{\text {exp }}(t)\right)\right|^{2} \mathrm{~d} t+\frac{1}{2}\left|\mathbf{R}\left(\mathbf{p}-\mathbf{p}_{0}\right)\right|^{2} \\
& -\int_{0}^{T} \mathcal{F}\left(\mathbf{y}(t), \mathbf{y}^{\prime}(t), \mathbf{p}, t\right)^{\mathrm{T}} \mathbf{z}(t) \mathrm{d} t-\mathbf{y}(0)^{\mathrm{T}} \mathbf{z}(0)
\end{aligned}
$$

where $(\mathbf{y}, \mathbf{p}, \mathbf{z})$ are considered independent and $(\cdot)^{\mathrm{T}}$ is the transposition. Minimizing the misfit function $\mathcal{J}(\mathbf{p})$ where $\mathbf{y}$ verifies (3) is then equivalent to writing the first-order stationarity conditions for $\mathcal{L}(\mathbf{y}, \mathbf{p}, \mathbf{z})$. This can be clearly seen when expressing the first-order stationarity condition with respect to $\mathbf{z}$ :

$$
-\int_{0}^{T} \mathcal{F}\left(\mathbf{y}(t), \mathbf{y}^{\prime}(t), \mathbf{p}, t\right)^{\mathrm{T}} \delta \mathbf{z}(t) \mathrm{d} t-\mathbf{y}(0)^{\mathrm{T}} \delta \mathbf{z}(0)=0
$$

for any admissible $\delta \mathbf{z}(t)$. This expression is actually the weak formulation of the state equation (3).

\subsection{Adjoint state problem}

When writing the first-order stationarity condition for $\mathcal{L}(\mathbf{y}, \mathbf{p}, \mathbf{z})$ with respect to $\mathbf{y}$, the following relation is obtained:

$$
\begin{array}{r}
\int_{0}^{T} \delta \mathbf{y}(t)^{\mathrm{T}}\left(\mathbf{A}^{\mathrm{T}} \mathbf{A}\left(\mathbf{y}(t)-\mathbf{y}_{\text {exp }}(t)\right)\right) \mathrm{d} t \\
-\int_{0}^{T}\left(\nabla_{\mathbf{y}} \mathcal{F} \delta \mathbf{y}(t)+\nabla_{\mathbf{y}^{\prime}} \mathcal{F} \delta \mathbf{y}^{\prime}(t)\right)^{\mathrm{T}} \mathbf{z}(t) \mathrm{d} t-\delta \mathbf{y}(0)^{\mathrm{T}} \mathbf{z}(0)=0
\end{array}
$$

for any $\delta \mathbf{y}(t)$ verifying null initial conditions. $\nabla_{\mathbf{y}} \mathcal{F}$ and $\nabla_{\mathbf{y}^{\prime}} \mathcal{F}$ stand for the directional derivatives of $\mathcal{F}$ with respect to $\mathbf{y}$ and $\mathbf{y}^{\prime}$ respectively. After integrating by parts, the previous weak formulation leads to the following expression:

$$
\begin{array}{r}
\int_{0}^{T} \delta \mathbf{y}(t)^{\mathrm{T}}\left(\mathbf{A}^{\mathrm{T}} \mathbf{A}\left(\mathbf{y}(t)-\mathbf{y}_{\text {exp }}(t)\right)\right) \mathrm{d} t \\
-\int_{0}^{T} \delta \mathbf{y}(t)^{\mathrm{T}}\left(\nabla_{\mathbf{y}} \mathcal{F}^{\mathrm{T}} \mathbf{z}(t)-\left(\nabla_{\mathbf{y}^{\prime}} \mathcal{F}^{\mathrm{T}} \mathbf{z}(t)\right)^{\prime}\right) \mathrm{d} t-\delta \mathbf{y}(T)^{\mathrm{T}} \nabla_{\mathbf{y}^{\prime}} \mathcal{F}^{\mathrm{T}} \mathbf{z}(T)=0
\end{array}
$$


which is equivalent to the following strong formulation defining the adjoint state problem:

$$
\begin{aligned}
& \nabla_{\mathbf{y}} \mathcal{F}^{\mathrm{T}} \mathbf{z}(t)-\left(\nabla_{\mathbf{y}^{\prime}} \mathcal{F}^{\mathrm{T}} \mathbf{z}(t)\right)^{\prime}=\mathbf{A}^{\mathrm{T}} \mathbf{A}\left(\mathbf{y}(t)-\mathbf{y}_{\text {exp }}(t)\right) \quad \forall t \in[0, T] \\
& \nabla_{\mathbf{y}^{\prime}} \mathcal{F}^{\mathrm{T}} \mathbf{z}(T)=\mathbf{0}
\end{aligned}
$$

The adjoint state problem is classical [16] and consists in solving for $\mathbf{z}(t)$ a linear time-backward ODE along with final conditions.

\subsection{Optimality conditions for the parameters}

Once the adjoint state problem is solved, the gradient of the misfit function with respect to $\mathbf{p}$ can be evaluated. Because of the equivalence between the constrained minimization of $\mathcal{J}(\mathbf{p})$ and the stationarity of $\mathcal{L}(\mathbf{y}(t ; \mathbf{p}), \mathbf{p}, \mathbf{z}(t))$, it can be written that:

$$
\nabla_{\mathbf{p}} \mathcal{J}(\mathbf{p})=\nabla_{\mathbf{p}} \mathcal{L}(\mathbf{y}(t ; \mathbf{p}), \mathbf{p}, \mathbf{z}(t))=\mathbf{R}^{\mathrm{T}} \mathbf{R}\left(\mathbf{p}-\mathbf{p}_{0}\right)-\int_{0}^{T} \nabla_{\mathbf{p}} \mathcal{F}^{\mathrm{T}} \mathbf{z}(t) \mathrm{d} t
$$

and should be equal to zero at optimality. This is equivalent to say that the optimal parameters depend on a weighted time average of the derivative of the state equation (3) with respect to the parameters vector $\mathbf{p}$, multiplied by the solution of the adjoint state problem (9).

The minimization problem eventually consists in solving three ODEs with unknowns $(\mathbf{y}(t), \mathbf{z}(t), \mathbf{p})$ : the forward problem (3), the adjoint problem (9) and the optimality equation (10) set equal to zero. The straightforward solution is not possible here, as the adjoint problem is time-backward: a staggered process is then proposed instead, where the forward and adjoint problems are solved successively on the one hand, and the optimality equation on the other hand is used to derive successive estimates of the misfit function gradient. These estimates are used in a classical gradient-based optimization algorithm, such as a line-search or a trust-region method. In addition, for the solution of the adjoint state problem, a change of variable for the time is first introduced in order to solve a time-forward problem along with initial conditions, rather than the original time-backward problem.

In terms of computational cost, evaluating the gradient of the misfit function is cheaper and more robust using the adjoint state formulation than with a finite difference formula. Whenever evaluating the gradient associated with $P$ parameters, the finite difference formula requires the solutions of $P$ additional forward problems, each one being evaluated with the same parameters 
as the computed forward problem, except for one parameter which is slightly perturbed in order to derive the corresponding component of the misfit function gradient. In addition, the computation of an integral is required for each of these $P$ forward solutions, in order to evaluate the associated misfit function.

On the contrary, when using the adjoint state formulation, only one additional ODE (9) has to be solved, and since this ODE is formally close to the forward problem, the solution of the adjoint state problem has the same cost as the solution of the forward problem. Then the computation of an integral is needed for each component of the gradient. To conclude, for each step of the staggered process, only two ODEs have to be solved and $N$ integrals have to be evaluated when using the adjoint state formulation, whereas $P+1$ ODE solutions and $N$ integrals evaluations are required with the finite difference formula.

\section{Parameter identification of the RT model}

\subsection{Formulation of the identification problem}

The parameter identification is achieved according to the formulation presented in the previous section. Similarly to (4), the misfit function quantifies the discrepancy between the computed and measured forces when a specific displacement is prescribed to the bushing:

$$
\begin{aligned}
\mathcal{J}(\mathbf{p}) & =\frac{1}{2} \int_{0}^{T}\left|K_{0} y_{0}(t)+K_{p a}\left(y_{0}(t)-y_{1}(t ; \mathbf{p})\right)-F_{\text {exp }}(t)\right|^{2} \mathrm{~d} t \\
& +\frac{1}{2}\left|\mathbf{R}\left(\mathbf{p}-\mathbf{p}_{0}\right)\right|^{2}
\end{aligned}
$$

where $F_{\text {exp }}(t)$ is the measured force, $\mathbf{R}=\operatorname{diag}\left(R_{K_{0}}, R_{K_{p a}}, R_{K_{r}}, R_{C_{0}}, R_{\alpha}\right)$ comprises the different regularization parameters to be set, and typical values for

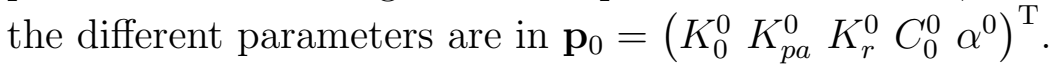

The adjoint state formulation used for evaluating the misfit function gradient consists in solving the following time-backward equations:

$$
\begin{gathered}
\left(K_{p a}+K_{r}\right) z_{1}(t)-K_{r} z_{2}(t)-C_{0} \alpha\left|y_{1}^{\prime}(t ; \mathbf{p})\right|^{\alpha-1} z_{1}^{\prime}(t) \\
-C_{0} \alpha(\alpha-1) \operatorname{sign}\left(y_{1}^{\prime}(t ; \mathbf{p})\right) y_{1}^{\prime \prime}(t ; \mathbf{p})\left|y_{1}^{\prime}(t ; \mathbf{p})\right|^{\alpha-2} z_{1}(t)=-K_{p a}\left(F(t ; \mathbf{p})-F_{e x p}(t)\right) \\
\vdots \\
-K_{r} z_{k-1}(t)+2 K_{r} z_{k}(t)-K_{r} z_{k+1}(t)-C_{0} \alpha\left|y_{k}^{\prime}(t ; \mathbf{p})\right|^{\alpha-1} z_{k}^{\prime}(t)
\end{gathered}
$$




$$
\begin{gathered}
-C_{0} \alpha(\alpha-1) \operatorname{sign}\left(y_{k}^{\prime}(t ; \mathbf{p})\right) y_{k}^{\prime \prime}(t ; \mathbf{p})\left|y_{k}^{\prime}(t ; \mathbf{p})\right|^{\alpha-2} z_{k}(t)=0 \\
\vdots \\
-K_{r} z_{N-1}(t)+2 K_{r} z_{N}(t)-C_{0} \alpha\left|y_{N}^{\prime}(t ; \mathbf{p})\right|^{\alpha-1} z_{N}^{\prime}(t) \\
-C_{0} \alpha(\alpha-1) \operatorname{sign}\left(y_{N}^{\prime}(t ; \mathbf{p})\right) y_{N}^{\prime \prime}(t ; \mathbf{p})\left|y_{N}^{\prime}(t ; \mathbf{p})\right|^{\alpha-2} z_{N}(t)=0
\end{gathered}
$$

for $1<k<N$, with vanishing final conditions, and where the force applied on the bushing, as evaluated by the RT model, is $F(t ; \mathbf{p})=K_{0} y_{0}(t)+K_{p a}\left(y_{0}(t)-\right.$ $\left.y_{1}(t ; \mathbf{p})\right)$. The gradient of the misfit function then comprises all the following partial derivatives:

$$
\begin{aligned}
& \nabla_{K_{0}} \mathcal{J}=\int_{0}^{T} y_{0}(t)\left(F(t ; \mathbf{p})-F_{\text {exp }}(t)\right) \mathrm{d} t+R_{K_{0}}^{2}\left(K_{0}-K_{0}^{0}\right) \\
& \nabla_{K_{p a}} \mathcal{J}=\int_{0}^{T}\left(y_{0}(t)-y_{1}(t ; \mathbf{p})\right)\left(F(t ; \mathbf{p})-F_{\text {exp }}(t)+z_{1}(t)\right) \mathrm{d} t+R_{K_{p a}}^{2}\left(K_{p a}-K_{p a}^{0}\right) \\
& \nabla_{K_{r}} \mathcal{J}=-\int_{0}^{T}\left(y_{1}(t ; \mathbf{p})-y_{2}(t ; \mathbf{p})\right) z_{1}(t) \mathrm{d} t-\int_{0}^{T}\left(-y_{N-1}(t ; \mathbf{p})+2 y_{N}(t ; \mathbf{p})\right) z_{N}(t) \mathrm{d} t \\
& -\sum_{k=2}^{N-1} \int_{0}^{T}\left(-y_{k-1}(t ; \mathbf{p})+2 y_{k}(t ; \mathbf{p})-y_{k+1}(t ; \mathbf{p})\right) z_{k}(t) \mathrm{d} t+R_{K_{r}}^{2}\left(K_{r}-K_{r}^{0}\right) \\
& \nabla_{C_{0}} \mathcal{J}=-\sum_{k=1}^{N} \int_{0}^{T} \operatorname{sign}\left(y_{k}^{\prime}(t ; \mathbf{p})\right)\left|y_{k}^{\prime}(t ; \mathbf{p})\right|^{\alpha} z_{k}(t) \mathrm{d} t+R_{C_{0}}^{2}\left(C_{0}-C_{0}^{0}\right) \\
& \nabla_{\alpha} \mathcal{J}=-\sum_{k=1}^{N} \int_{0}^{T} C_{0} \operatorname{sign}\left(y_{k}^{\prime}(t ; \mathbf{p})\right) \ln \left|y_{k}^{\prime}(t ; \mathbf{p}) \| y_{k}^{\prime}(t ; \mathbf{p})\right|^{\alpha} z_{n}(t) \mathrm{d} t+R_{\alpha}^{2}\left(\alpha-\alpha^{0}\right)
\end{aligned}
$$

This computed gradient is to be used within two classical minimization algorithms: a line-search method and a trust-region method [17]. The first results, however, are very unsatisfactory: the computed gradient is clearly erroneous, and can even, in some cases, return infinite values. Further investigations allowed to determine that this came from the viscoelastic law (1) used in the RT model: depending on the value of $\alpha$ and due to the occurrences of the sign and absolute value functions, this law can be nearly discontinuous, so that its derivative with respect to the velocity can be extremely high for very small velocities. Furthermore, since $\alpha<1$, the adjoint problem (12) is singular when one of the velocities $y_{k}^{\prime}$ is equal to zero. This analysis is corroborated by the results of [18], which shows that it is impossible to derive the correct misfit function gradients when discontinuities occur 
in the equations to solve, with errors of a magnitude independent of the time step size. The authors then propose to smooth the discontinuities, and show that the correct gradient values can be evaluated when the time step size is 'sufficiently small' in comparison with the smoothing parameter.

Therefore, in order to smooth the solved equations, we replaced the occurrences of the sign and absolute value functions by the following expressions respectively:

$$
\begin{aligned}
\operatorname{sign}_{s}\left(y^{\prime}\right) & =\frac{2}{1+\mathrm{e}^{-s y^{\prime}}}-1 \\
\left|y^{\prime}\right|_{s} & =\frac{2}{s} \ln \left(1+\mathrm{e}^{s y^{\prime}}\right)-y^{\prime}
\end{aligned}
$$

where $s$ is a constant to set: the lower $s$ is, the smoother the functions sign and $|\cdot|_{s}$ are. The first function is actually a classical sigmoid function, and the second one is the primitive function of the first one: this allows to directly replace the sign and absolute value functions by their smoothed counterparts in Equations (2), (12) and (13). The influence of the smoothing parameter $s$ is depicted in Figure 10. To determine the best value for $s$, the accuracy of the adjoint state solution has to be determined.
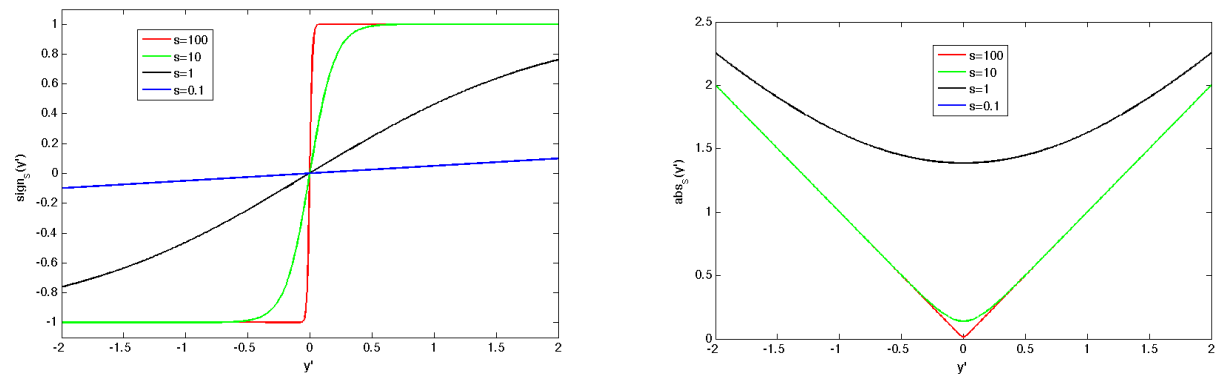

Figure 10: Influence of the smoothing parameter $s$ : smoothed sign function (left) and smoothed absolute value function (right)

\subsection{Validation of the adjoint state solution}

A classical way to check the accuracy of the computed adjoint state is to use the mathematical property of the adjoint state equation (9), whose 
operator $\mathcal{G}$ should be the adjoint of the operator associated with the differentiated forward state equation $\nabla_{\mathbf{y}} \mathcal{F}$. The idea is to solve the following equations, for any arbitrary vector a:

$$
\begin{aligned}
\nabla_{\mathbf{y}} \mathcal{F} \mathbf{b} & =\mathbf{a} \\
\mathcal{G} \mathbf{c} & =\mathbf{b}
\end{aligned}
$$

Since theoretically one has $\nabla_{\mathbf{y}} \mathcal{F}^{\mathrm{T}}=\mathcal{G}$, the following equality should hold, provided the adjoint state has been correctly solved:

$$
\mathbf{a}^{\mathrm{T}} \mathbf{c}=\mathbf{b}^{\mathrm{T}} \mathbf{b}
$$

This dot-product test should be verified for different values of a to be positively sure that the adjoint state solution is correctly calculated.

A series of numerical tests were conducted in order to determine the most relevant value that should be chosen for the smoothing parameter $s$. Using an arbitrary set of parameter values $\mathbf{p}_{\text {exp }}=\left(K_{0}^{e x p} K_{p a}^{\text {exp }} K_{r}^{\text {exp }} C_{0}^{e x p} \alpha^{e x p}\right)^{\mathrm{T}}$ in Equation (2) to simulate an experimental reference, the misfit function (with no regularization term) and its gradient are evaluated for a given set of parameters whose values are quite different from the experimental ones: $\left(K_{0}^{e x p} / 22 K_{p a}^{e x p} K_{r}^{e x p} / 2 C_{0}^{e x p} / 22 \alpha^{e x p}\right)^{\mathrm{T}}$. The results in the case of different values of $s$ (as well as without any smoothing) are listed in Table 1. In addition, the dot-product test (16) has been applied for $\mathbf{a}=(1 \cdots 1)^{\mathrm{T}}$ and the associated results are listed in Table 1 as well.

\begin{tabular}{c||c|c|c|c|c|c|c|c|c} 
Smoothing & $\mathcal{J}$ & $\nabla_{K_{0}} \mathcal{J}$ & $\nabla_{K_{p a}} \mathcal{J}$ & $\nabla_{K_{r}} \mathcal{J}$ & $\nabla_{C_{0}} \mathcal{J}$ & $\nabla_{\alpha} \mathcal{J}$ & $\mathbf{a}^{\mathrm{T}} \mathbf{c}$ & $\mathbf{b}^{\mathrm{T}} \mathbf{b}$ \\
\hline$s=0.1$ & 921787.149 & -4752.446 & -16.3299 & -277.728 & 49467.59 & -13149.70 & 0.021569 & 0.021576 \\
$s=1$ & 888192.640 & -4665.399 & -17.630 & -268.873 & 67022.15 & -13154.49 & 0.021378 & 0.021351 \\
$s=10$ & 888192.739 & -4665.399 & -17.735 & -285.087 & 38570.69 & -11927.34 & 0.028785 & 0.020740 \\
$s=100$ & 888192.736 & -4665.399 & $-5.6 \cdot 10^{9}$ & $-8.2 \cdot 10^{9}$ & $-1.3 \cdot 10^{13}$ & $1.2 \cdot 10^{12}$ & $>3 \cdot 10^{7}$ & 0.020359 \\
none & 888192.736 & -4665.399 & infinite & infinite & infinite & infinite & infinite & 0.020245
\end{tabular}

Table 1: Evaluation of the misfit function and its gradient, and dot-product test for different smoothing settings

As predicted, the dot-product test is more and more accurately verified as the smoothing increases, that is as $s$ decreases. Other values of a give similar results. The best value for the smoothing seems to be $s=1$, for it represents a trade-off between the correct estimation of the misfit function $\mathcal{J}$ (and of its gradient) and the validation of the adjoint state characterized by the dot-product test. 
In Table 2, we compare the directional derivatives previously computed with $s=1$ with those estimated with a finite-difference formula and no smoothing. Even if the estimates are of the same order of magnitude, some differences can be noticed, excepted for $\nabla_{K_{0}} \mathcal{J}$ where $\mathbf{z}$ is not used. On the one hand, the computed problems are slightly different because of the smoothing; on the other hand, the estimates which are computed using a finite-difference formula without smoothing can not be considered as references, for they depend on the discretisation step used in the formula and the smaller this discretisation step, the stronger the impact of the tolerance of the forward state solution on the result of the formula. All in all, using some smoothing and estimating the misfit function gradient by means of the adjoint state seems to be relevant to solve the identification problem, as can be seen in the following section. Eventually, the value $s=1$ is used in what follows.

\begin{tabular}{c|c|c|c|c|c} 
Method & $\nabla_{K_{0}} \mathcal{J}$ & $\nabla_{K_{p a}} \mathcal{J}$ & $\nabla_{K_{r}} \mathcal{J}$ & $\nabla_{C_{0}} \mathcal{J}$ & $\nabla_{\alpha} \mathcal{J}$ \\
\hline adjoint state with smoothing & -4665.399 & -17.630 & -268.873 & 67022.15 & -13154.49 \\
finite diff. without smoothing & -4665.399 & -6.7776 & -241.145 & 39672.53 & -8141.242
\end{tabular}

Table 2: Evaluation of the misfit function gradient

\subsection{Identification results with synthetic data}

In order to assess the accuracy of the identification process, preliminary tests using synthetic data are proposed. Since actual experimental data come from imposed quasistatic periodic inputs as well as Heaviside step functions, it seems relevant to study similar inputs to quantify the accuracy of the identification results. The final choice here is to compute the response to sinusoidal displacements, and use them as synthetic data for the identification process. Figure 6 (left) shows the typical curves that can be obtained for such inputs. Synthetic data are computed using an arbitrary set of parameter values $\mathbf{p}_{\text {exp }}=\left(K_{0}^{e x p} K_{p a}^{e x p} K_{r}^{e x p} C_{0}^{e x p} \alpha^{e x p}\right)^{\mathrm{T}}$ in the forward problem (2), for a sinusoidal displacement input of $1 \mathrm{~Hz}$-frequency.

Two different gradient-based algorithms, proposed in the Optimisation Toolbox for Matlab, are tested: a line-search method, consisting in a QuasiNewton method with the BFGS formula for the approximation of the Hessian matrix [17], and a trust-region method, based on the interior reflective Newton method [19]. Identification results for both methods and close initial 
values $\left(K_{0}^{\text {exp }} 4 K_{p a}^{e x p} / 32 K_{r}^{\text {exp }} / 32 C_{0}^{\text {exp }} 2 \alpha^{e x p}\right)^{\mathrm{T}}$ are listed in Table 3. They range from satisfactory to excellent depending on the parameter, and tend to be better with the trust-region method.

\begin{tabular}{c|c|c|c|c|c} 
Method & $\frac{K_{0}}{K_{0}^{\exp }}$ & $\frac{K_{p a}}{K_{p a}^{e x p}}$ & $\frac{K_{r}}{K_{r}^{\text {exp }}}$ & $\frac{C_{0}}{C_{0}^{e x p}}$ & $\frac{\alpha}{\alpha^{e x p}}$ \\
\hline line-search & 1.099 & 1.095 & 0.649 & 0.972 & 1.524 \\
trust-region & 1.001 & 0.975 & 0.767 & 1.270 & 0.922
\end{tabular}

Table 3: Identification results for synthetic data and initial values close to the 'experimental' ones

In order to check the robustness of the identification process, initial values much farther from the values used for computing the synthetic data are proposed: $\left(2 K_{0}^{\exp } 5 K_{p a}^{\exp } K_{r}^{\exp } / 5 C_{0}^{e x p} / 55 \alpha^{e x p}\right)^{\mathrm{T}}$. They lead to the results listed in Table 4. The results are far worse than in the case of initial values chosen close to the 'experimental' ones. This means that there are many local minima in the misfit function, making the identification process difficult as is. In addition, some parameters, such as $C_{0}$ and $\alpha$, seem not to have a strong impact on the response, for the bushing is loaded in a quasi-static way. Despite this final remark, it can be concluded that, between the two methods, the trust-region method gives the best results.

\begin{tabular}{c|c|c|c|c|c} 
Method & $\frac{K_{0}}{K_{0}^{e x p}}$ & $\frac{K_{p a}}{K_{p a}^{e x p}}$ & $\frac{K_{r}}{K_{r}^{e x p}}$ & $\frac{C_{0}}{C_{0}^{e x p}}$ & $\frac{\alpha}{\alpha^{e x p}}$ \\
\hline line-search & 1.328 & 5.121 & 0.227 & 0.498 & 7.954 \\
trust-region & 1.300 & 2.960 & 0.361 & 0.499 & 4.884
\end{tabular}

Table 4: Identification results for synthetic data and initial values far from the 'experimental' ones

To go further, some regularization is then required. It is not, however, straightforward when dealing with the actual experimental data: since the model uses rheological elements, there are no typical values which can easily be chosen for the vector $\mathbf{p}_{0}=\left(K_{0}^{0} K_{p a}^{0} K_{r}^{0} C_{0}^{0} \alpha^{0}\right)^{\mathrm{T}}$. Moreover, there is actually no quantitative information available concerning the measurement noise corrupting the experimental data. Applying principles such as Morozov's [20] in order to set relevant values for the regularization parameters contained in $\mathbf{R}$ is then not really possible. With these two drawbacks, the 
resulting regularization may not be relevant; therefore, rather than applying a classical Tikhonov's regularization in the identification process, a technique is proposed in the following section to give a first guess of the initial values to be used in the minimization algorithm.

\subsection{Determination of relevant initial values for the identification process}

Due to the ill-posedness of the identification problem, it is crucial to be able to give relevant initial values to the minimization algorithm in order not to converge to a local minimum which would lie too far from the global one. This can be done by using the STS model described in Section 3.1 rather than the RT model, because the STS model can be analytically inverted under some simple assumptions. Starting from the experimental hysteresis curve, it is possible to get values for the associated parameters $\mathbf{p}_{S T S}=\left(\begin{array}{llll}K_{0}^{S} & K_{p a}^{S} & K_{r}^{S} & F_{f}\end{array}\right)^{\mathrm{T}}$. Then, initial values for the RT model's parameters can be derived from these parameter values.

First, it is assumed that all the STS cells but one (i.e. $N-1$ cells) are loaded when the hysteresis curve is obtained: this is equivalent to assume that the bushing is loaded with a sufficiently high-amplitude displacement. In this case, three representative features of the hysteresis curve can be determined as functions of the STS parameters $\mathbf{p}_{S T S}=\left(\begin{array}{lll}K_{0}^{S} K_{p a}^{S} K_{r}^{S} F_{f}\end{array}\right)^{\mathrm{T}}$ :

- the secant stiffness associated with the two extremities of the hysteresis cycle (with $N$ odd):

$$
K_{\mathrm{sec}}=K_{0}^{S}+\frac{K_{p a}^{S} K_{r}^{S}}{\frac{N-1}{2} K_{p a}^{S}+K_{r}^{S}}
$$

- the slope of the line being tangent to the hysteresis cycle just after a cusp:

$$
T_{\mathrm{g} 1}=K_{0}^{S}+K_{p a}^{S}
$$

- the slope of the line being tangent to the hysteresis cycle just before a cusp:

$$
T_{\mathrm{g} 2}=K_{0}^{S}+\frac{K_{p a}^{S} K_{r}^{S}}{(N-1) K_{p a}^{S}+K_{r}^{S}}
$$


540 541

These features are summarized on Figure 11. Finally, the maximal force $F_{\max }$ applied on the bushing when $N-1$ STS cells are loaded can be written as follows:

$$
F_{\max }=K_{0}\left(\frac{F_{f} N(N-1)}{K_{r}^{S}}+\frac{2 F_{f} N}{K_{p a}^{S}}\right)+2 N F_{f}
$$

This maximal force is obtained for a displacement amplitude $X_{\max }$, which means that $F_{\max }=K_{\mathrm{sec}} X_{\max }$.

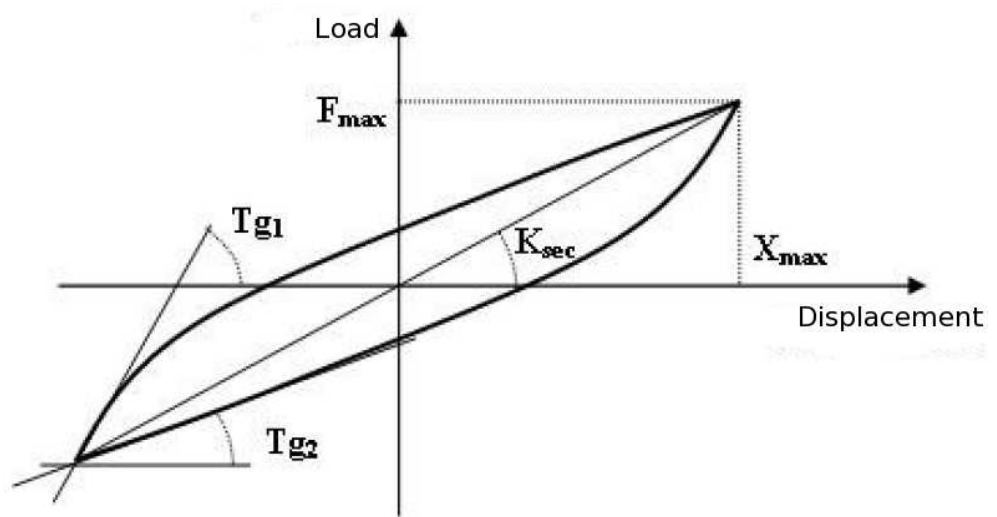

Figure 11: Features of the hysteresis curve associated with the STS model

$$
\text { par }
$$

parameters $\mathbf{p}_{S T S}=\left(\begin{array}{llll}K_{0}^{S} & K_{p a}^{S} & K_{r}^{S} & F_{f}\end{array}\right)^{\mathrm{T}}$, for example in terms of slopes before and after a cusp, and maximal force and displacement :

$$
\begin{aligned}
K_{0}^{S} & =\frac{F_{\max }\left(T_{\mathrm{g} 1}+T_{\mathrm{g} 2}\right)-2 T_{\mathrm{g} 1} T_{\mathrm{g} 2} X_{\max }}{2 F_{\max }-\left(T_{\mathrm{g} 1}+T_{\mathrm{g} 2}\right) X_{\max }} \\
K_{p a}^{S} & =\frac{\left(T_{\mathrm{g} 1}-T_{\mathrm{g} 2}\right)\left(F_{\max }-T_{\mathrm{g} 1} X_{\max }\right)}{2 F_{\max }-\left(T_{\mathrm{g} 1}+T_{\mathrm{g} 2}\right) X_{\max }} \\
K_{r}^{S} & =-\frac{(N-1)\left(T_{\mathrm{g} 1}-T_{\mathrm{g} 2}\right)\left(F_{\max }-T_{\mathrm{g} 1} X_{\max }\right)\left(F_{\max }-T_{\mathrm{g} 2} X_{\max }\right)}{\left(2 F_{\max }-\left(T_{\mathrm{g} 1}+T_{\mathrm{g} 2}\right) X_{\max }\right)^{2}} \\
F_{f} & =\frac{\left(F_{\max }-T_{\mathrm{g} 1} X_{\max }\right)\left(F_{\max }-T_{\mathrm{g} 2} X_{\max }\right)}{N\left(2 F_{\max }-\left(T_{\mathrm{g} 1}+T_{\mathrm{g} 2}\right) X_{\max }\right)}
\end{aligned}
$$

If it is assumed that the STS and RT models are roughly similar, the first three expressions can give straightforward initial values for the first three RT 
model's parameters:

$$
K_{0}^{0}=K_{0}^{S} \quad K_{p a}^{0}=K_{p a}^{S} \quad K_{r}^{0}=K_{r}^{S}
$$

In order to determine estimates for the last two, it is assumed that the viscoelastic part (1) of the RT cell should be analogous to the threshold force $F_{f}$ of the STS cell when the bushing is loaded at a (mean) speed $V_{0}$, that is:

$$
C_{0}^{0}=\frac{F_{f}}{V_{0}^{\alpha^{0}}}
$$

To remain quite close to the solid friction behaviour of the STS cell, an arbitrary choice of a small value for $\alpha^{0}$ is made, such as one tenth or one fifth.

This strategy has been tested on the example from the previous section. In Table 5 are listed the initial values of the RT model's parameters that are obtained from the synthetic hysteresis cycle when two different values of $\alpha^{0}$ are proposed. The obtained values show some discrepancies with the 'experimental' ones: it can be explained by the fact that the slopes $T_{g 1}$ and $T_{g 2}$ cannot be determined without some error and that any inaccuracy in the associated values can have a strong impact on the determination of the STS model's parameter values. As an example of this sensitivity, Figure 12 shows how the first three STS model's parameters evolve as the measured value of the slope $T_{g 2}$ varies: the changes can go up to nearly $100 \%$ for a $10 \%$-change in the value $T_{g 2}$. As any other inverse problem, the quality of the inversion of the formulas associated with the STS model shows a strong dependence on the noise in the measurements.

\begin{tabular}{c||c|c|c|c}
$\alpha^{0}$ & $\frac{K_{0}^{0}}{K_{0}^{e x p}}$ & $\frac{K_{p a}^{0}}{K_{p a}^{e x p}}$ & $\frac{K_{r}^{0}}{K_{r}^{e x p}}$ & $\frac{C_{0}^{0}}{C_{0}^{e x p}}$ \\
\hline $1 / 10$ & 0.770 & 1.105 & 1.468 & 0.703 \\
$1 / 5$ & 0.770 & 1.105 & 1.468 & 0.740
\end{tabular}

Table 5: Initial RT model's parameter values using an STS model identification and the synthetic hysteresis cycle

All these results, however, show that it is possible to get reasonable initial values for the RT model's parameters: all the proposed values lie in a range of the same magnitude as the one proposed in the first identification example with synthetic data (with associated results in Table 3). Moreover, the 


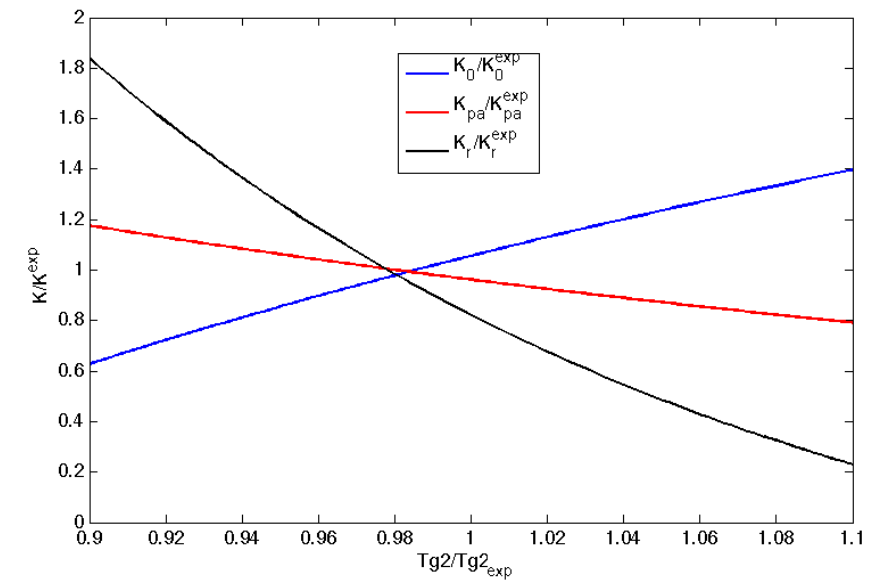

Figure 12: Normalized values of the first three STS model's parameters with respect to the slope $T_{g 2}$ measured on the hysteresis curve

arbitrary choice of a value for $\alpha^{0}$ does not seem to have too high an impact on the proposed value for $C_{0}^{0}$. Finally, since all these values are perturbed by some noise, it seems better, as stated previously, to use them as initial values for the minimization algorithm rather than nominal values in a Tikhonov's regularization term such as the one proposed in (11). The resulting benefit should be the minimization algorithm to likely start in the attraction basin of the global minimum of the misfit function.

\subsection{Identification results from actual data}

As described previously, actual experimental data come from quasistatic periodic as well as fast transitory loads, applied to the bushing by means of a specific test rig. Two simple test configurations are proposed: the first one consists in applying to the bushing a triangular periodic displacement at a rather limited speed and is used in the identification process. The second configuration consists in applying a displacement characterised by a Heaviside step function to observe relaxation in the bushing (the input being not truly discontinuous, but being characterised by a 0.1 s time interval of increase): but rather than using the associated measurements in the identification process as well, one prefers to keep them as a further reference in order to validate the identified model a posteriori. 
The same gradient-based algorithms as in Section 5.3 are used here. Initial values of the RT model's parameters are given according to the strategy described in Section 5.4; since the experimental curve (depicted in Figure 13 or 14) is not perfectly symmetric, an average value of each feature has been used. Table 6 lists the (relative) identified values of the RT model's parameters as well as a normalized value of the discrepancy between the model's results and experimental data: this value is calculated as the square root of the final misfit function divided by a norm $\mathcal{J}_{0}$ of the experimental data, defined by the following expression:

$$
\mathcal{J}_{0}=\frac{1}{2} \int_{0}^{T}\left|F_{\text {exp }}(t)\right|^{2} \mathrm{~d} t
$$

This normalized value is a good guess of the relative identification error. Whereas this error was equal to $15.32 \%$ with the proposed initial values, it drops significantly for the values identified with the two methods. Eventually, the two methods lead to very similar results, even if the line-search method seems to provide a slightly better solution.

\begin{tabular}{c||c|c|c|c|c||c} 
Method & $\frac{K_{0}}{K_{0}^{0}}$ & $\frac{K_{p a}}{K_{p a}^{0}}$ & $\frac{K_{r}}{K_{r}^{0}}$ & $\frac{C_{0}}{C_{0}^{0}}$ & $\frac{\alpha}{\alpha^{0}}$ & $\sqrt{\frac{\mathcal{J}}{\mathcal{J}_{0}}}$ \\
\hline line-search & 1.217 & 1.049 & 1.088 & 0.930 & 1.197 & $3.11 \%$ \\
trust-region & 1.182 & 1.145 & 1.156 & 0.889 & 1.153 & $3.20 \%$
\end{tabular}

Table 6: Identification results for actual data (triangular periodic displacement)

Figures 13 and 14 (left) show for each method the comparison between the experimental data and the responses associated with the initial RT model and with the identified RT model. As seen previously with the relative errors, the agreement is very good in the case of the triangular periodic loading. Let us note that only the stabilised hysteresis curves are recorded in these quasistatic tests: no information is available on the first loading of the bushing. This latter is mandatory in the numerical simulation: it is introduced by considering that a linearly-varying displacement is applied to the bushing: afterwards, the stabilised hysteresis cycle is obtained. The misfit function is based on these experimental and predicted stabilised cycles only.

Validation of these results is possible with the experimental response to the Heaviside step function, which has not been used in the identification 
process. Figures 13 and 14 (right) show the good agreement between the response associated with the identified RT model and the experimental data. Since these latter have not been used in the identification process, this result shows the relevance of the RT model as well as the quality of the identification of the associated parameters. In particular, both the relaxation phenomenon and the asymptotic steady-state load are well described by the identified model, when compared with the experimental data.
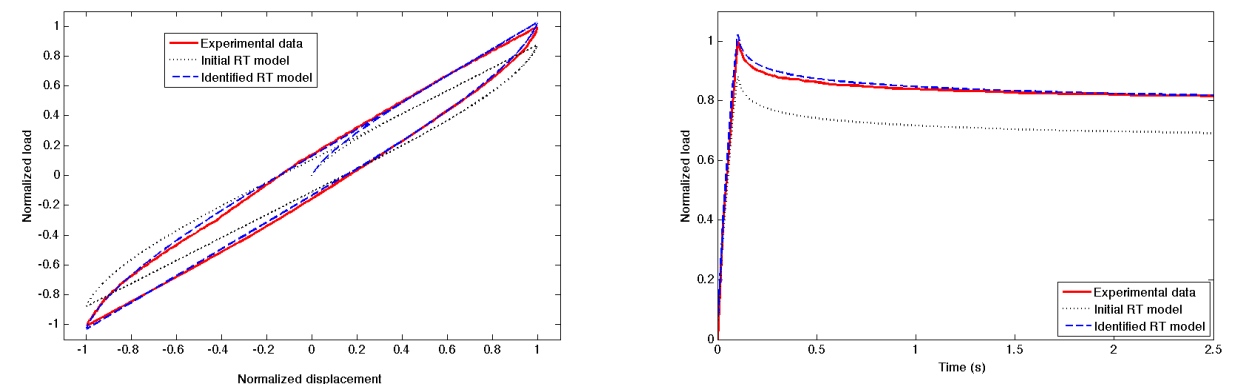

Figure 13: Identification results with the line-search method: comparison with the experimental data used (left) and with experimental data not used in the identification process (right)
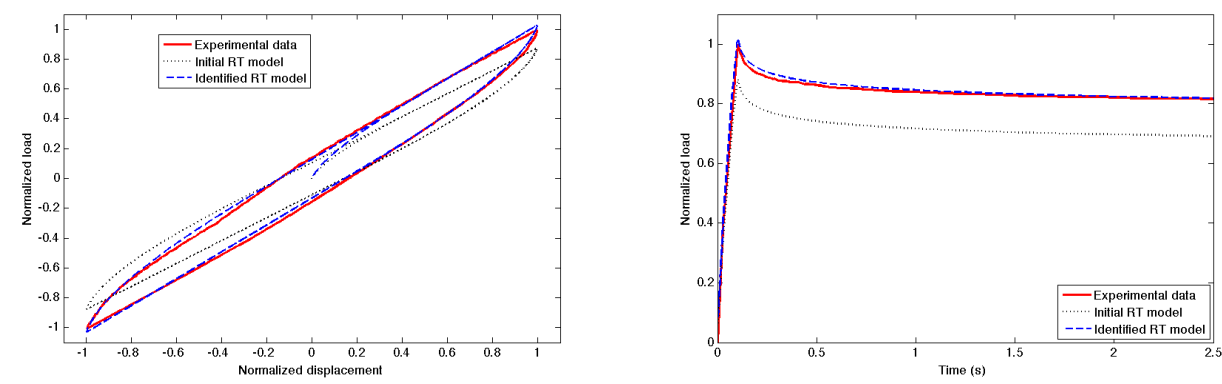

Figure 14: Identification results with the trust-region method: comparison with the experimental data used (left) and with experimental data not used in the identification process (right) 


\section{Conclusion}

Here a strategy for identifying the parameters of a nonlinear time-dependent model has been presented and applied on the example of a rubber bushing typically used in car chassis. The proposed model is based on components exhibiting a viscoelastic behaviour which can tend towards the description of contact-type phenomena. The identification has been achieved in the time domain using an adjoint state formulation allowing an efficient estimation of the misfit function's gradient.

It was shown that the near-discontinuous behaviour of the proposed model can lead to numerical difficulties, which may prevent the model's parameters to be correctly identified due to an inaccurate estimation of the adjoint state variable. The solution has consisted in smoothing the discontinuities by means of sigmoid functions: a trade-off allowed to correct the estimates of the adjoint state without any significant loss of accuracy for the forward state solution.

The relevance of the proposed model as well as the robustness of the method have been shown with the parameter identification from actual data: in addition, the obtained results have been validated with experimental information which was not used in the identification process. Such an identification allows to deal with accurate models, which are simple enough to be implemented in multibody simulation softwares, allowing to study wellknown phenomena occurring in a typical vehicle chassis.

In a more general way, the strategy presented here can be applied to other time-dependent problems with contact-type phenomena, and should allow to achieve the parameter identification both accurately and efficiently.

\section{Acknowledgements}

The authors would like to acknowledge the support of PSA Peugeot Citroën for this study.

\section{References}

[1] A. Lion, A constitutive model for carbon black filled rubber: experimental investigations and mathematical representation, Continuum Mechanics and Thermodynamics 6 (1996) 153-169. 
[2] C. Miehe, J. Keck, Superimposed finite elastic-viscoelastic-plastoelastic stress response with damage in filled rubbery polymers. Experiments, modelling and algorithmic implementation, Journal of the Mechanics and Physics of Solids 48 (2000) 323-365.

[3] P. Haupt, K. Sedlan, Viscoplasticity of elastomeric materials: experimental facts and constitutive modelling, Archive of Applied Mechanics 71 (2001) 89-109.

[4] A. R. Payne, A note on the existence of a yield point in the dynamic modulus of loaded vulcanizates, Journal of Applied Polymer Science $3(7)(1960) 127$.

[5] L. Mullins, Softening of rubber by deformation, Rubber Chemistry and Technology Journal 42 (1969) 339-362.

[6] V. A. Coveney, D. E. Johnson, D. M. Turner, A triboelastic model for the cyclic mechanical behavior of filled vulcanizates, Rubber Chemistry and Technology Journal 68 (1995) 660-670.

[7] V. A. Coveney, D. E. Johnson, D. M. Turner, Rate-dependent modeling of a highly filled vulcanizate, Rubber Chemistry and Technology Journal 73 (4) (2000) 565-577.

[8] U. Tornar, Application of strain integral damping to engine mounts, in: P. Sas, M. De Mucnck (Eds.), ISMA 2006 - International Conference on Noise and Vibration Engineering, Leuven, Belgium, 2006, pp. CDROM.

[9] R. L. Bagley, P. J. Torvik, Fractional calculus - a different approach to the analysis of viscoelastically damped structures, AIAA Journal 21 (1983) 741-748.

[10] M. Sjöberg, L. Kari, Nonlinear isolator dynamics at finite deformations: an effective hyperelastic, fractional derivative, generalized friction model, Nonlinear Dynamics 33 (2003) 323-336.

[11] J.-F. Deü, D. Matignon, Simulation of fractionally damped mechanical systems by means of a Newmark-diffusive scheme, Computers and Mathematics with Applications 59 (2010) 1745-1753. 
[12] R. Bouc, Modèle mathématique d'hystérésis, Acustica 24 (1971) 16-25.

[13] Y. K. Wen, Method of random vibration of hysteretic systems, ASCE Journal of the Engineering Mechanics Division 102 (1976) 249-263.

[14] Y. Q. Ni, J. M. Ko, C. W. Wong, Identification of non-linear hysteretic isolators from periodic vibration tests, Journal of Sound and Vibration 217 (4) (1998) 737-756.

[15] J. K. Ok, W. S. Yoo, J. H. Sohn, New nonlinear bushing model for general excitations using Bouc-Wen hysteretic model, International Journal of Automotive Technology 9 (2) (2008) 183-190.

[16] A. Constantinescu, N. Tardieu, On the identification of elastoviscoplastic constitutive laws from indentation tests, Inverse Problems in Science and Engineering 9 (1) (2001) 19-44.

[17] J. Nocedal, S. J. Wright, Numerical Optimization, 2nd Edition, Springer, 2006.

[18] D. E. Stewart, M. Anitescu, Optimal control of systems with discontinuous differential equations, Numerische Mathematik 114 (4) (2010) 653-695.

[19] T. F. Coleman, Y. Li, On the convergence of reflective Newton methods for large-scale nonlinear minimization subject to bounds, Mathematical Programming 67 (2) (1994) 189-224.

[20] V. A. Morozov, Methods for solving incorrectly posed problems, Springer, 1984. 